\title{
The effect of nitro-oleic versus losartan in diabetic nephropathy: modulation of parathyroid hormone-related protein
}

\author{
A.S. Mohammed ${ }^{1}$, N.B. Sadek ${ }^{1}$, M.A. Eshra' ${ }^{1}$, E.A.H. Khowailed'1, L.A. Rashed², B.E. Aboulhoda ${ }^{3}$ \\ ${ }^{1}$ Department of Physiology, Faculty of Medicine, Cairo University, Egypt \\ 2Department of Biochemistry, Faculty of Medicine, Cairo University, Egypt \\ ${ }^{3}$ Department of Anatomy and Embrology, Faculty of Medicine, Cairo University, Egypt
}

[Received: 3 May 2018; Accepted: 18 May 2018]

Background: Parathyroid hormone-related protein (PTHrP) involvement in the mechanisms related to angiotensin II (AngII)-induced renal injury has become an emerging concern. The current study was thus designed to compare the possible preventive and therapeutic effect of Angll antagonists, losartan and nitro-oleic (NO2-OA) acid, on diabetic nephropathy (DN) and evaluate their effect on PTHrP modulation as well as on the functional and histopathological parameters in the kidney of diabetic rats.

Materials and methods: Forty eight adult male Sprague Dawley rats were divided into control group, DN group, pre-diabetic nephropathy (pre-DN) losartan group, pre-diabetic nephropathy nitro-oleic acid (pre-DN NO2-OA) group, post-diabetic nephropathy (post-DN) losartan and post-diabetic nephropathy nitro-oleic acid (post-DN NO2-OA) groups. At the end of the study, systolic blood pressure (SBP), serum fasting glucose, glomerular filtration rate (GFR), urea, urea albumin excretion (UAE), serum angiotensin, renal PTHrP gene expression and correlations between PTHrP and SBP, serum glucose, Angll and kidney functions were evaluated. Histological examination, Masson's trichrome, periodic acid-Schiff staining as well as morphometric analysis and histopathological scoring for tubular and glomerular parameters have been carried out.

Results: Prophylactic losartan and NO2-OA were associated with improvement in SBP, serum glucose, urea, GFR, UAE, with reduction in serum Angll and PTHrP overexpression observed in diabetic kidney. Treatment with losartan and NO2-OA showed the same effect except that post-DN NO2-OA showed no significant effect regarding kidney function. Strong correlations were observed between PTHrP and SBP, serum glucose, Angll and kidney functions. Histopathological results revealed obvious improvement in glomerulosclerosis, vascular and tubular injury parameters in prophylactic groups especially with losartan.

Conclusions: Both pre and post-DN losartan, NO2-OA may have a potential role in protection and regression of DN through reduction of PTHrP overexpression. (Folia Morphol 2019; 78, 1: 79-90)

Key words: diabetic nephropathy, losartan, nitro-oleic acid, angiotensin II, parathyroid hormone related protein 


\section{INTRODUCTION}

The treatment guidelines for diabetic nephropathy (DN) always focus on slowing the progression of nephropathy by controlling glucose levels and decreasing blood pressure. However, this remains always suboptimal. Thus, it's become more important to focus on novel molecular mechanisms and treatment modalities [21]. One of those newly evolving lines is the renal interaction between the reninangiotensin-aldosterone system (RAS), parathyroid hormone related protein (PTHrP) and angiotensin II (Angll) in the kidney [13]. Current concepts indicate that PTHrP is a regulating factor, being more similar to other known growth factors and cytokines than to parathyroid hormone [3].

Based on the role of the RAS as crucial pathway involved in pathogenesis and progression of $\mathrm{DN}$, along with the previous studies demonstrating interaction between PTHrP and Angll in the kidney [13, 25], heart [19] and in vascular smooth muscle cells $[14,16]$ the current study aimed at evaluating the effect Angll antagonists in an experimental model of DN using one pharmacological drug, losartan as an angiotensin receptor blocker (ARB), and a non-pharmacological agent, nitro-oleic acid (olive oil), guided by the recent recommendations provided by American Diabetic Association regarding the value of ARBs in DN [21].

Losartan is a reversible, competitive inhibitor of the AT1 receptor (AT1R) while nitro oleic acid (NO2$-\mathrm{OA}$ ) binds directly to the AT1R without interfering with Angll binding, suggesting that molecular interactions between NO2-OA and AT1R are likely to differ from ARBs competitive inhibition [27].

The current study was designed to evaluate the possible preventive and therapeutic effects of losartan and NO2-OA on the functional and histopathological parameters of DN and to elucidate the effect of those drugs on PTHrP modulation in an attempt to provide appropriate ground for better management of DN.

\footnotetext{
MATERIALS AND METHODS

Materials

Chemicals were purchased from Sigma (St. Louis, MO, USA). (1) Streptozotocin (STZ) powder, dissolved in fresh $0.05 \mathrm{M}$ citrate buffer, $\mathrm{pH}=4.5$ and immediately injected intraperitoneally (i.p.) as recommended by the National Institute of Diabetes Animal Models of Diabetic Complications Consortium. (2) Losartan $10 \mathrm{mg} / \mathrm{kg}$ dissolved in $1 \mathrm{~mL}$ saline in oral gavage [12].
}

(3) 10-Nitro oleic acid was infused at $5 \mathrm{mg} / \mathrm{kg} /$ day via osmotic minipump [12].

\section{Experimental animals}

Forty-eight adult male Sprague Dawley rats (150$200 \mathrm{~g}$ ) were purchased from the animal care unit. All experimental procedures were carried out according to guidelines for care and use of laboratory animals published by the United States National Institute of Health (NIH 1985). The study was approved by Kasr al-ainy Ethical Committee, Faculty of Medicine, Cairo University. Animals had free access to food and water.

\section{Preparation of diabetic rat}

The animals were injected with STZ $(50 \mathrm{mg} / \mathrm{kg}$, i.p.) [7]. Five days after injection, the rats with fasting blood glucose higher than $200 \mathrm{mg} / \mathrm{dL}$ were considered diabetic and were used for the experiment which lasted for 8 weeks.

\section{Animal groups}

Animals were divided into the following groups 8 rats each:

- Control group;

- Diabetic nephropathy (DN) group: Diabetic rats that received equal amount of vehicle. The estimated duration for developing nephropathy is 4 weeks after induction of diabetes [20]. Nephropathy was confirmed by significant increase in proteinuria and serum creatinine;

- Pre-DN losartan group (losartan prophylactic group): received losartan $10 \mathrm{mg} / \mathrm{kg}$ dissolved in $1 \mathrm{~mL}$ saline in oral gavage [12], as prophylaxis to DN, starting from the onset of diabetes, and continued for 4 weeks (the estimated time for occurrence of nephropathy) and then the rats were left without any medications till the end of study;

- Pre-DN NO2-OA (NO2-OA prophylactic group): received NO2-OA infused at $5 \mathrm{mg} / \mathrm{kg} /$ day via osmotic minipump, as prophylaxis to DN in the same fashion as the losartan prophylactic group;

- Post-DN losartan (losartan treatment group): received losartan $10 \mathrm{mg} / \mathrm{kg}$ dissolved in $1 \mathrm{~mL}$ saline in oral gavage [12] for 4 weeks, started after the occurrence of diabetic nephropathy till the end of the study;

- Post-DN NO2-OA (NO2-OA treatment group): received $\mathrm{NO2}-\mathrm{OA}$ infused at $5 \mathrm{mg} / \mathrm{kg} /$ day via osmotic minipump for 4 weeks, started after the 
occurrence of diabetic nephropathy till the end of the study.

\section{Experimental design}

The animals were daily observed for signs of morbidity and mortality. There was neither mortality nor changes in food and water consumption and health status in the studied animals. At the end of the study, all rats were subjected to overnight fasting; blood samples were collected from the retro-orbital plexus for assessment of fasting serum glucose level, blood urea nitrogen (BUN), serum angiotensin and plasma creatinine for assessing glomerular filtration rate (GFR). Urine samples were collected for measuring creatinine in urine and $24 \mathrm{~h}$ urine were collected, for measuring GFR and urinary albumin excretion (UAE). Systolic blood pressure (SBP) in rats was measured. Finally, kidney tissue samples were extracted for histopathological and quantitative real-time polymerase chain reaction (PCR) gene expressions of PTHrP.

\section{Measurement of SBP}

Using the Power lab data acquisition system (AD instruments; Pty Ltd., Australia); an electronic version of the traditional sphygmomanometer cuff method.

\section{Biochemical assessment}

Measurement of fasting serum glucose. The glucose was measured colorimetrically after oxidation of glucose. The absorbance of standard and samples were measured spectrophotometrically against blank at $500 \mathrm{~nm}$ wavelength.

Determination of kidney functions. (1) Measurement of UAE using Albuwell M Kit: Murine Microalbuminuria ELISA. Before sampling, rats were deprived from food for 1 day with free access to water. (2) Measurement of serum creatinine and BUN using kits (Bioclin, Santa Coloma, Spain) with absorbance at $340 \mathrm{~mm}$ based on the reaction between ammonia and alpha-ketoglutaric acid when urea is hydrolysed. (3) Determination of GFR was done by calculating the creatinine clearance: Creatinine clearance $=$ (urine level of creatinine $\times 24 \mathrm{~h}$ urine volume $) \div$ (plasma level of creatinine $\times 24 \times 60$ ).

Measurement of angiotensin II enzyme. Using enzyme Immunoassay (EIA) Kit (RayBio ${ }^{\circledR}$ ).

Quantitative analysis of gene expression of PTHrP by real time PCR. Total RNA was extracted from kidney tissue homogenate using Qiagen tissue extraction kit (Qiagen, USA) according to manufacturer's instruction. Complementary DNA was synthesized from $1 \mu \mathrm{g}$ RNA using SuperScript III First-Strand Synthesis System as described in the manufacturer's protocol (\#K1621, Fermentas, Waltham, MA, USA).

Real-time PCR amplification and analysis were performed using an Applied Biosystem with software version 3.1 (StepOne ${ }^{\mathrm{TM}}$, USA). The reaction contained gene-specific primer pairs for PTHrP with following sequence Sense 5-TGGTGTTCCTGCTCAGCTA-3, antisense 5-CCTCGTCGTCTGACCCAAA-3 and $\beta$-actin sense: 5'-CAG GAT GGC GTG AGG GAG AGC-3', antisense: 5'- , 5'-AAG GTG TGA TGG TGG GAA TGG-3'-. Relative expression of gene mRNA was calculated. All values were normalised to $\beta$-actin which was used as the control housekeeping gene and reported as fold change over background levels.

\section{Histopathological examination}

Kidneys from all groups were dissected and fixed immediately in $10 \%$ formol saline. Then the specimens were washed, dehydrated in ascending grades of ethanol, cleared in xylene and impregnated in paraffin. Sections of $5 \mu \mathrm{m}$ thickness were cut and subjected to haematoxylin and eosin (H\&E), Masson's trichrome (MT) and periodic acid-Schiff (PAS) staining.

\section{Quantitative morphometric analysis}

All histomorphometric parameters were estimated via the Leica image analyser software programme (Leica Qwin 500C Ltd., Cambridge, England) in 10 non-overlapping randomly-selected fields.

For H\&E-stained sections. The measured parameters were Bowman's spaces, glomerular areas, tubular diameter, tubular epithelial height and vascular congestion area per cent.

For MT-stained sections. The area per cent of collagen fibres was measured using the measuring field menu; the area and area percentage were masked by blue binary colour and estimated using the image analyser.

For PAS-stained sections. Length of PCT brush border and PAS optical density were assessed.

Histopathology scoring. Histopathology scoring was carried out using a scoring system reflecting the grading of tubular necrosis and cast formation in H\&E stained sections as previously described [4]: 0 (none), 1 (< 10\%), 2 (10 - < 25\%), 3 ( $25-<45 \%)$, 4 (45 - < 75\%), and 5 (> 75\%). 


\section{Statistical methods}

Data were analysed using statistical package SPSS version 24. Data were summarised using mean and standard deviation. Comparisons between groups were done using ANOVA with multiple comparisons Bonferroni post hoc testing. Correlations between variables were done using Pearson correlation coefficients. P-values $<0.05$ were considered statistically significant.

\section{RESULTS}

Changes in fasting serum glucose level

Induction of diabetes resulted in significant increase in serum glucose level. Serum glucose levels in pre-DN losartan and pre-DN (NO2-OA) groups were significantly reduced compared to diabetic group. However, prophylactic losartan showed significant improving compared to prophylactic NO2-OA (Fig. 1A).

Post-DN losartan and post-DN (NO2-OA) group showed significant decline in serum glucose, with no significant difference in relation to each other.

\section{Results of SBP}

Systolic blood pressure level was significantly increased in diabetic rats. Prophylactic losartan and NO2-OA resulted in significant improvement of SBP, with much more improvement with losartan (Fig. 1B). Treatment of diabetic rats with either losartan or NO2-OA showed significant reduction in SBP.

\section{Results of kidney function parameters:} UEA, GFR, and urea

Kidney function parameters were significantly deteriorated in diabetic rats relative to control rats with increase in urea and UAE and decrease in GFR (Fig. 1C-E).

Prophylactic losartan showed partial protection from DN presented by significant decline in urea and UAE and significant rise to GFR. Similarly, prophylactic NO2-OA showed significant improvement in these parameters. Losartan treatment showed significant lower urea and UAE and higher GFR.

\section{Changes in serum angiotensin and PTHrP expression in renal tissue}

Serum angiotensin and PTHrP expression showed a significant increase in diabetic rats (Fig. 1F). After prophylactic administration of both losartan and NO2-OA, serum angiotensin level decreased significantly and PTHrP expression recovered significantly.
Regarding the treatable effect of both drugs, the reduced levels of serum angiotensin and PTHrP expression were significantly improved compared to diabetic rats. Only angiotensin level showed significant difference in post-DN losartan relative to post-DN NO2-OA group with non-significant increase in PTHrP.

Correlation studies showed that PTHrP showed a strong positive correlation with angiotensin, urea and UAE and negative correlation with GFR as shown in Figure 2.

\section{Histopathological results}

Examination of H\&E stained sections (Fig. 1H, l; 3). Examination of the DN group revealed pleopathological glomerular and tubular changes with statistically significant deterioration in tubular necrosis and cast formation. Comparing the protective role of losartan and NO2-OA demonstrated that pre-DN losartan resulted in preservation of glomerular and tubular architectural patterns while NO2-OA showed only partial conservation. Losartan or NO2-OA treatment after DN slightly improved glomerulosclerosis.

Assessment of vasculopathy in H\&E stained sections (Fig. 3). The DN group exhibited severely dilated congested blood vessels surrounded by hyalinosis. After prophylactic administration of either losartan or NO2-OA, marked statistically significant improvement in vascular congestion occurred with only few congested blood capillaries and minimal extravasation still noticed in the pre-DN NO2-OA group. Vascular congestive features on the other hand showed only minimal amelioration in both post DN treated losartan and NO2-OA groups.

Examination of PAS stained sections (Fig. 4). Diabetic rats displayed features of glomerulosclerosis manifested by excessive mesangial matrix deposition causing obliteration of the capillary lumina. Glomerular tuft adhesion, Bowman's capsule thickening and loss of the apical brush border of renal tubules were also predominant features in the DN group. Prophylactic losartan resulted in preservation of the tubular brush border with normal tubular basal laminae, while prophylactic NO2-OA showed only partial protection with areas of normal PAS reaction and others of focal thickening. Administration of either losartan or NO2-OA after induction of DN showed persistence of Bowman's capsule and capillary tuft basement membrane thickening in the losartan group and failure to restore the degenerated tubular brush border in the NO2-OA group. 
A

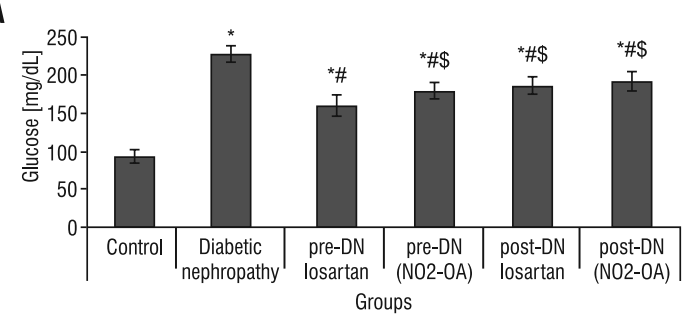

C

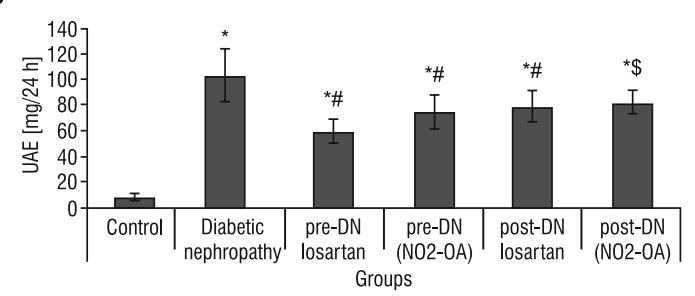

$\mathbf{E}$

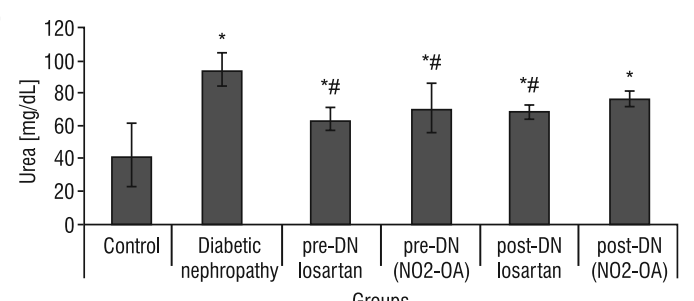

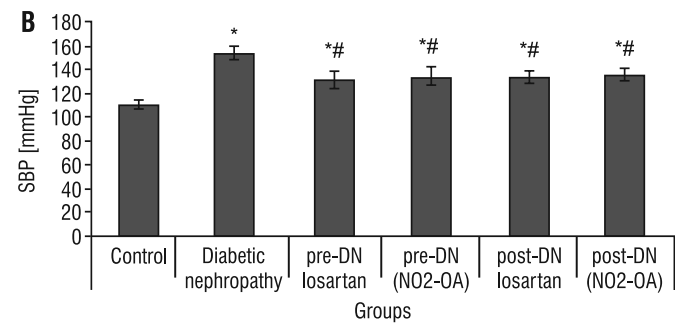

D
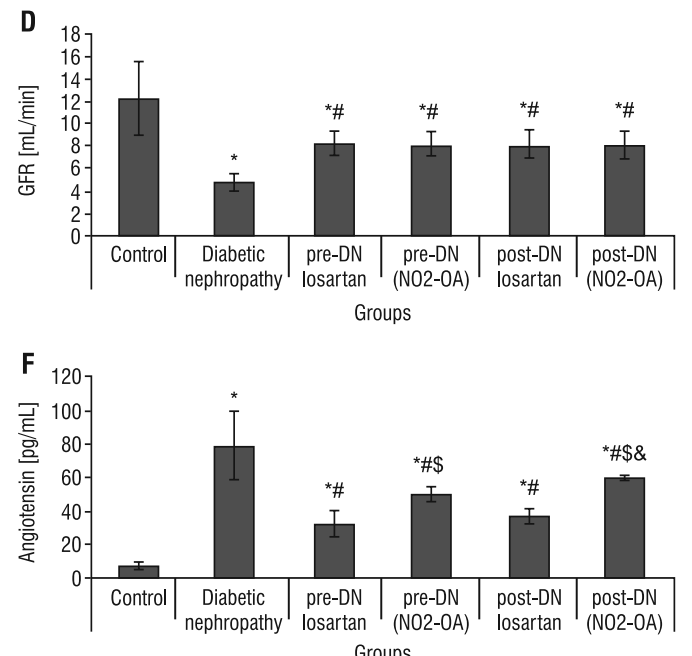
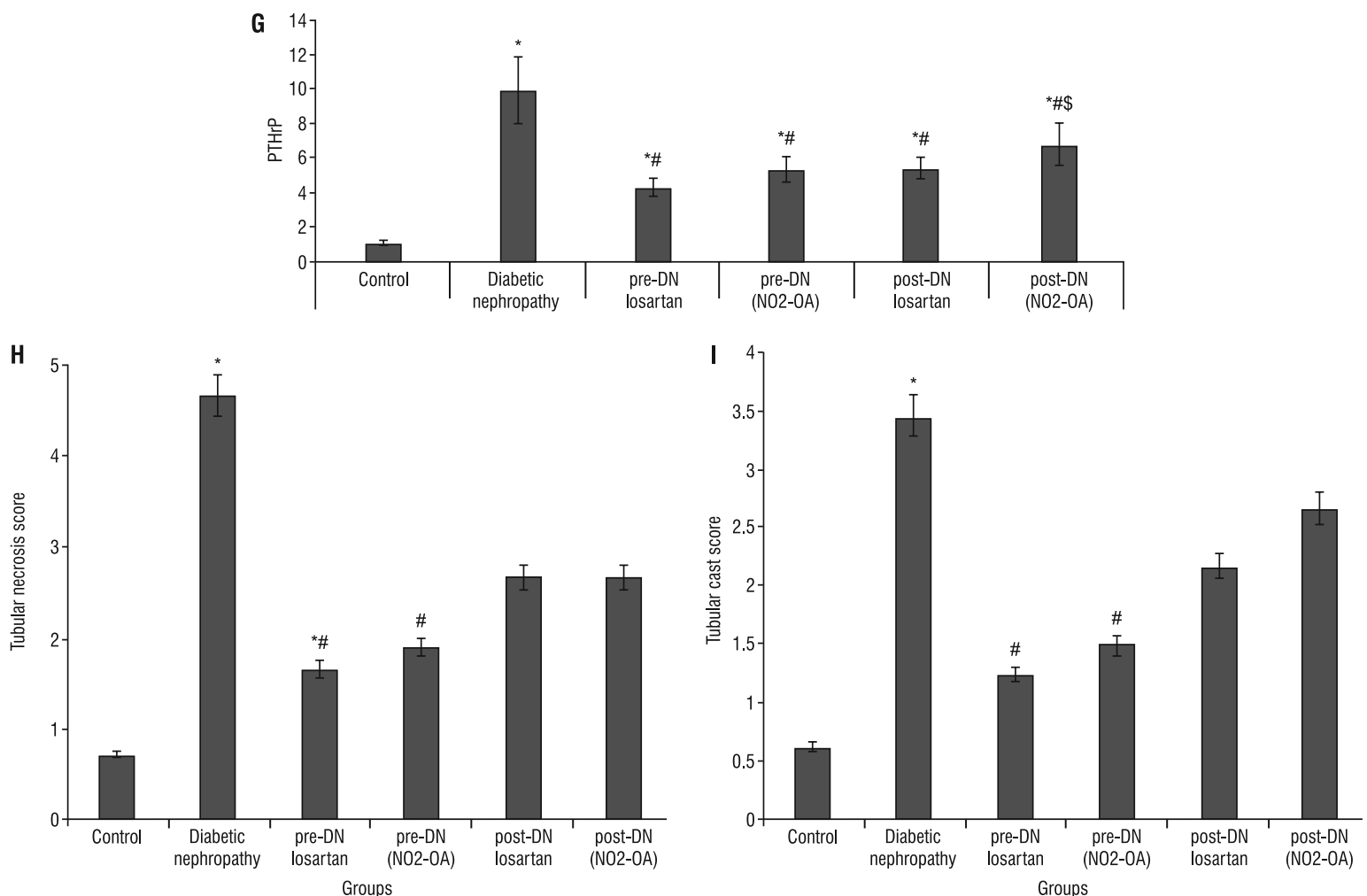

Figure 1. A. Fasting serum glucose; B. Systolic blood pressure (SBP); C. Albumin in urine (urine albumin excertion [UAE]); D. Glomerular filtration rate (GFR); E. Urea; F. Serum angiotensin; G. Parathyroid hormone related protein (PTHrP) expression in renal tissue among studied groups; H. Tubular necrosis score; I. Tubular cast score. Each vertical bar represents the mean \pm standard deviation of 10 animals; *Statistically significant compared to control group; \#Statistically significant compared to DN group; \$Statistically significant compared to pre-DN losartan group; \&Statistically significant compared to post-DN losartan group; DN — diabetic nephropathy; NO2-0A — nitro-oleic acid; pre-DN — pre-diabetic nephropathy; pre-DN N02-OA — pre diabetic nepropathy nitro-oleic acid; post-DN — post-diabetic nephropathy; post-DN NO2-AO — post diabetic nepropathy nitro-oleic acid. 

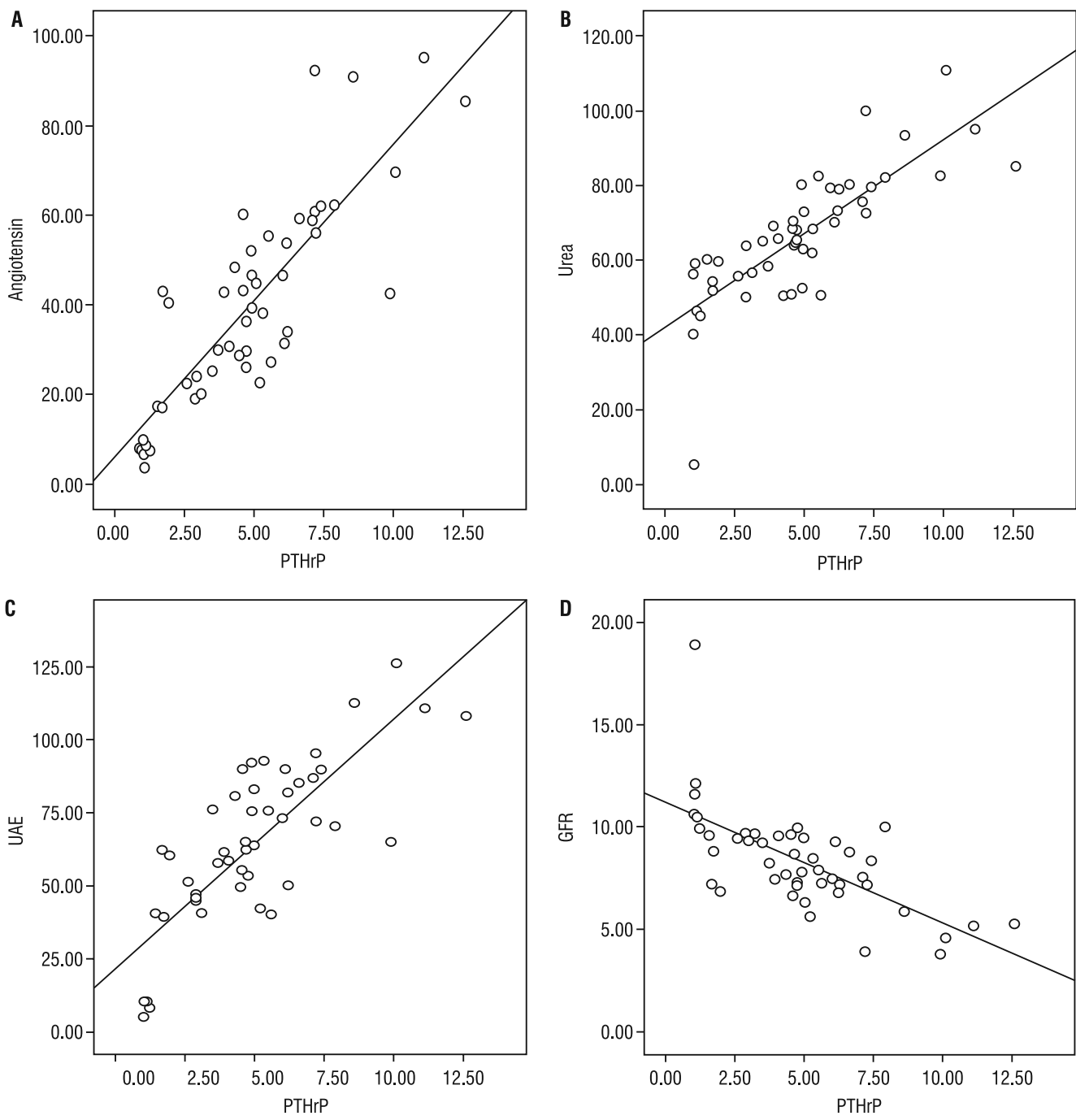

Figure 2. Parathyroid hormone related protein (PTHrP) correlation studies showing a strong positive correlation with PTHrP and angiotensin (A), urea (B) and urinary albumin excretion (UAE) (C) and negative correlation with glomerular filtration rate (GFR) (D).

Examination of MT stained sections (Fig. 5). The DN group displayed features of diabetic glomerulosclerosis and tubulointerstitial fibrosis. The pre-DN losartan group showed normal collagen distribution pattern around renal tubules and in the glomeruli except for localised areas of tuft-to-capsule adhesions, while pre-DN NO2-OA group showed partial conservation of the capillary lumina with occasional focal collagen deposition. Losartan treatment resulted in recovery from the glomerular sclerotic pattern but with compensatory tuft hypertrophy while treatment with NO2-OA resulted in non-significant improvement in glomerulosclerosis.

\section{Histomorphometric parameters (Table 1)}

Assessment of glomerular parameters showed significant improvement with prophylactic losartan and NO2-OA while the treatment groups on the other hand showed non-significant differences relative to the diabetic group. Regarding tubular parameters, the tubular diameter and tubular epithelial height showed significant increase in pre-DN losartan, preDN NO2-OA, and post-DN losartan groups, while the bush border length demonstrated significant increase only with pre-DN losartan.

\section{DISCUSSION}

This study has been designed to compare the possible preventive and therapeutic effect of losartan and NO2-OA on DN, and to evaluate their effects on PTHrP modulation in the kidney of diabetic rats.

The DN group in the current study demonstrated various functional changes in the form of deterioration of renal functions and significant elevation of 

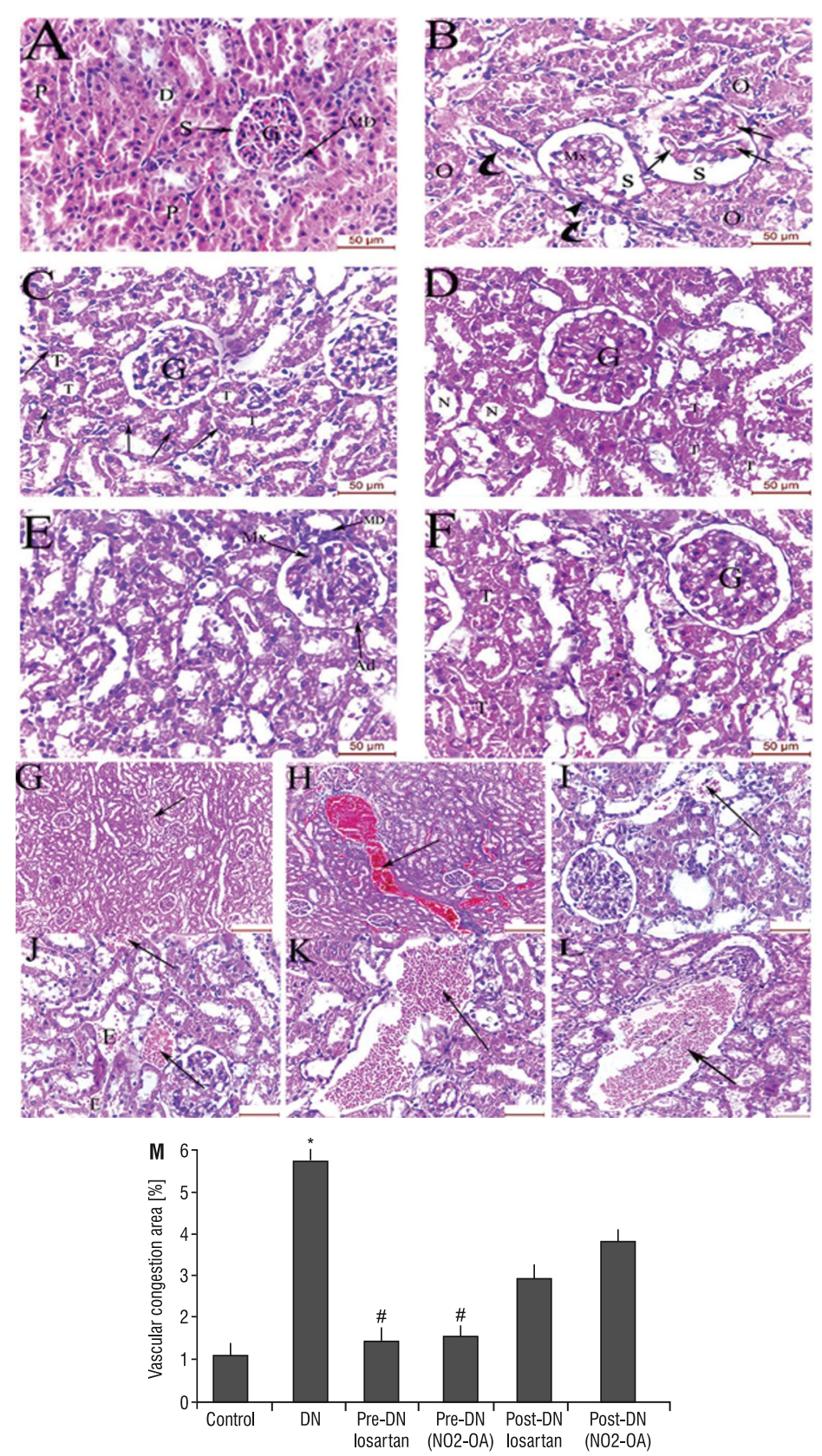

Figure 3. A. Control group showing normal renal corpuscle composed of a normal glomerulus $(\mathrm{G})$ formed of capillary tuft surrounded by Bowman's space (S); MD — macula densa; P — PCT; D — DCT; B. DN group showing mesangial matrix deposition (Mx), extra-glomerular mesangial expansion (arrow head), congestion of glomerular capillaries with occasional aneurysmal dilatation (arrows) and widening of urinary space (S) secondary to capillary tuft retraction. Some tubules show intraluminal exfoliation of epithelial cells (curved arrows); others appear obliterated $(0)$ with casts; C. Pre-DN losartan group showing preservation of the glomerular (G) architectural patterns. Most of the tubules (T) appear healthy with vesicular nuclei and prominent nucleoli (arrows); D. Pre-DN N02 group showing glomerulus (G) with moderately increased mesangial matrix. Some tubules (T) are degenerated, others appear normal (N); E. Post-DN losartan group showing glomerulus with tuft-to-capsule adhesion (Ad) (synechiae) and slight increase in mesangial matrix (Mx). Normal macula densa (MD) is observed; $\mathbf{F}$. Post-DN N02-OA group showing glomerulus $(G)$ with mesangial hypercelluarity. Few tubules $(T)$ appear filled with casts. H\&E $\times 400$; Scale bar $50 \mu$ m; G, H, I, J, K, L, M showing vascular changes. G. Control group showing normal vascular pattern; H. DN group showing severely dilated congested blood vessels (BV) surrounded by hyalinosis; I. Pre-DN losartan group showing obvious improvement of vascular congestion; J. Pre-DN NO2-OA group showing few congested blood vessels in addition to blood extravasation (E); K. Post-DN losartan group showing markedly dilated congested BV; L. Post-DN N02-OA group displaying obviously dilated congested BV in close vicinity of a glomerulus and surrounded by extra-glomerular mesangial cell expansion; M. Graph showing area per cent of vascular congestion in different groups; data are presented as mean \pm standard deviation ( $<0.05$ in DN vs. control, Pre-DN losartan vs. DN and Pre-DN N02-OA vs. DN); *Statistically significant compared to control, \#Statistically significant compared to DN; G, H: $\times 100$; Scale bar $200 \mu \mathrm{m}$; I, J, K: $\times 400$; Scale bar $50 \mu \mathrm{m}, \mathrm{L}: \times 200$; Scale bar $50 \mu \mathrm{m}$ ); DN — diabetic nephropathy; N02-OA — nitro-oleic acid; pre-DN — pre-diabetic nephropathy; pre-DN N02-0A — pre-diabetic nephropathy nitro-oleic acid; post-DN — post-diabetic nephropathy; post-DN N02-0A — post-diabetic nephropathy nitro-oleic acid. 

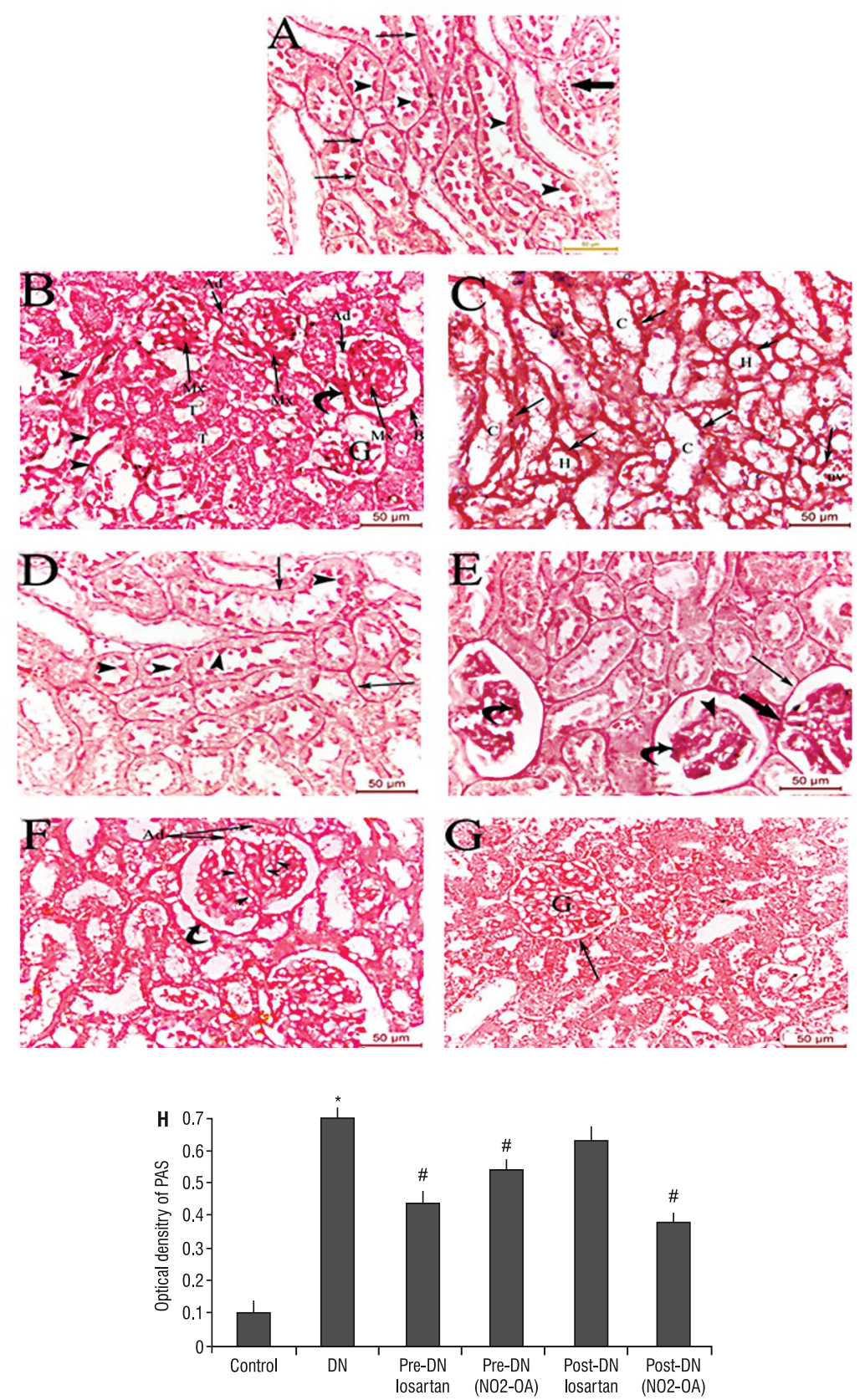

Figure 4. Periodic acid-Schiff (PAS) stained sections of: A. Control group showing positive PAS reaction in the intact apical brush border (arrow heads) and basement membrane of the renal tubules (thin arrows) and capillary basement membrane (thick arrows); B. DN group showing excessive mesangial matrix ( $\mathrm{Mx}$ ) deposition (Early stages of Kimmelstiel-Wilson lesion) causing obliteration of the capillary lumina and tuft adhesion (Ad) to Bowman's capsule. Areas of focal thickening of the parietal layer of Bowman's capsule (B), loss of the apical brush border of renal tubules (T), thickening of the basal membrane of macula densa (curved arrow) and walls of blood vessels (arrow heads) and a degenerated glomerulus (G); C. Diabetic rats adrenal medulla displaying thickening of the basement membrane (arrows) of collecting tubules (C), loop of Henle (H) and blood vessels (BV); D. Pre-DN losartan group showing restoration of tubular brush border (arrow heads) with normal thin tubular basal laminae (arrows); E. Pre-DN N02-0A group showing glomeruli with areas of normal PAS reaction (arrow heads), others with localised thickening (curved arrows). The Bowman's capsule shows few areas of focal thickening (thick arrow) and others of normal thickness (thin arrow); F. Post-DN losartan group showing thickened Bowman's capsule (curved arrow) and glomeruli with thickened capillary tuft basement membranes (arrow heads) with tuft-to-capsule adhesions (Ad); G. Post-DN NO2-OA group showing hypertrophied glomerulus (G) almost filling the Bowman's space and surrounded by thin corrugated Bowman's capsule (arrow). Most of the tubules exhibit degenerated brush border; H. Graph showing optical density of PAS in different groups; data are presented as mean \pm standard deviation $(p<0.05$ in DN vs. control, Pre-DN losartan vs. DN, Pre-DN N02-0A vs. DN, Post-DN losartan vs. DN and Post-DN N02-0A vs. DN; *Statistically significant compared to control; \#Statistically significant compared to DN; PAS $\times 400$; Scale bar $50 \mu \mathrm{m}$; DN — diabetic nephropathy; NO2-0A — nitro-oleic acid; pre-DN — pre-diabetic nephropathy; pre-DN N02-OA — pre-diabetic nephropathy nitro-oleic acid; post-DN — post-diabetic nephropathy; post-DN-NO2-OA — post-diabetic nephropathy nitro-oleic acid. 

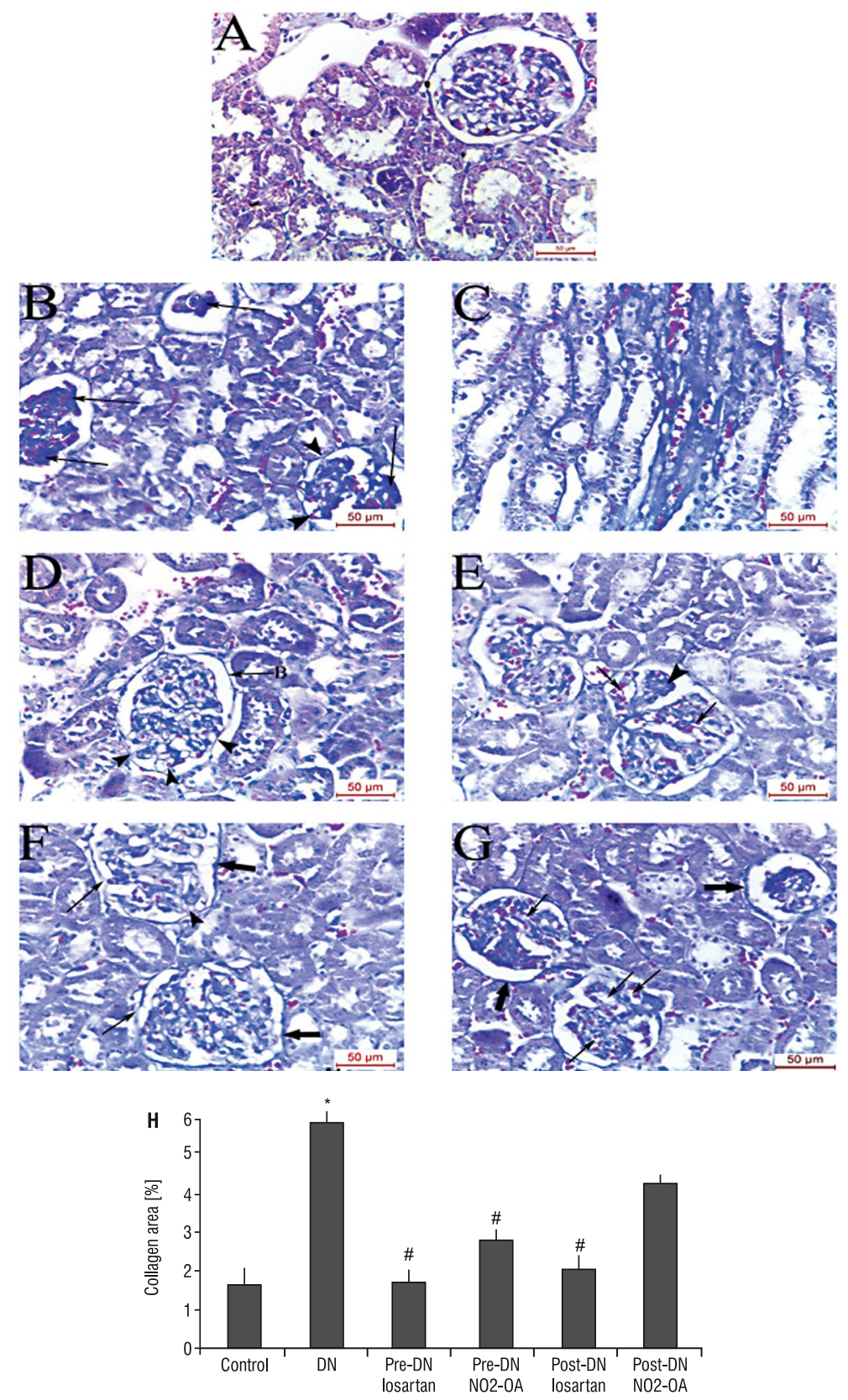

Figure 5. A. Control group demonstrating normal collagen distribution; B. DN group displaying sclerosed glomeruli with extensive collagen deposition (arrows) causing marked implosion of the capillary loops. Some glomeruli appear severely collapsed (C); others show tuft-to-capsule adhesion (arrow heads); C. DN group showing tubulointerstitial fibrosis; D. Pre-DN losartan group showing preservation of normal collagen distribution around renal tubules and in the glomeruli except for few localised areas of tuft-to-capsule adhesions (arrow heads). Thin but irregular Bowman's capsule (B) is observed; E. Pre-DN N02-OA group showing partial conservation of the capillary lumina (arrows) and focal area of increased collagen deposition (arrow head); F. Post-DN losartan group displaying recovery from the glomerular sclerotic pattern but with compensatory tuft hypertrophy and capsule adhesions (arrow head). Collagen bundles in the parietal layer of the Bowman's capsule show areas of thickening (thick arrow) as well as areas of rarefaction (thin arrow); G. Post-DN NO2-0A group showing few open capillary loops (arrows) in the sclerotic glomeruli and deposition of few thickened collagen bundles in the Bowman's capsule (arrow head); H. Graph showing area per cent of collagen deposition in different groups; data are presented as mean \pm standard deviation $(p<0.05$ in DN vs. control, Pre-DN losartan vs. DN, Pre-DN N02-OA vs. DN and Post-DN losartan vs. DN; *Statistically significant compared to control; \#Statistically significant compared to DN; Masson trichrome $\times 400$; Scale bar $50 \mu \mathrm{m}$; DN — diabetic nephropathy; N02-0A — nitro-0leic acid; pre-DN — pre-diabetic nephropathy; pre-DN N02-OA — pre-diabetic nephropathy nitro-oleic acid; post-DN — post-diabetic nephropathy; post-DN NO2-OA — post-diabetic nephropathy nitro-oleic acid. 
Table 1. Histomorphometric parameters

\begin{tabular}{|c|c|c|c|c|c|c|}
\hline & Control & DN & Pre-DN losatan & Pre-DN N02-0A & Post-DN losatan & Post-DN N02-0A \\
\hline Bowman's space $\left[\mu \mathrm{m}^{2}\right]$ & $1756.16 \pm 41.30$ & $\begin{array}{c}3007.50 \pm 15.14 \\
(p=0.007)^{* *}\end{array}$ & $\begin{array}{c}2053.06 \pm 66.01 \\
(p=0.024)^{*}\end{array}$ & $\begin{array}{c}2416.17 \pm 25.11 \\
(p=0.043)^{*}\end{array}$ & $\begin{array}{c}2524.12 \pm 81.31 \\
(p=0.051)^{N S}\end{array}$ & $\begin{array}{c}2991.57 \pm 39.18 \\
(p=1.65)^{\text {NS }}\end{array}$ \\
\hline Glomerular area $\left[\mu \mathrm{m}^{2}\right]$ & $3123.18 \pm$ & $\begin{array}{c}2001.61 \pm 54.02 \\
(p=0.042)^{*}\end{array}$ & $\begin{array}{c}2769.34 \pm 81.15 \\
(p=0.01)^{* *}\end{array}$ & $\begin{array}{c}2309.16 \pm 76.44 \\
(p=0.49)^{*}\end{array}$ & $\begin{array}{c}2187.16 \pm 46.02 \\
(p=0.66)^{\mathrm{Ns}}\end{array}$ & $\begin{array}{c}2052.75 \pm 71.09 \\
(p=3.02)^{\text {Ns }}\end{array}$ \\
\hline Tubular diameter $\left[\mu \mathrm{m}^{2}\right]$ & $39.87 \pm 2.79$ & $\begin{array}{l}22.28 \pm 3.1 \\
(p=0.021)^{*}\end{array}$ & $\begin{array}{l}33.12 \pm 4.07 \\
(p=0.032)^{*}\end{array}$ & $\begin{array}{l}27.63 \pm 5.61 \\
(p=0.041)^{*}\end{array}$ & $\begin{array}{l}29.88 \pm 2.09 \\
(p=0.037)^{*}\end{array}$ & $\begin{array}{l}25.18 \pm 1.49 \\
(p=0.07)^{\mathrm{NS}}\end{array}$ \\
\hline Tubular epithelial height $[\mu \mathrm{m}]$ & $19.72 \pm 2.66$ & $\begin{array}{l}8.84 \pm 3.19 \\
(p=0.036)^{*}\end{array}$ & $\begin{array}{l}15.63 \pm 2.44 \\
(p=0.012)^{*}\end{array}$ & $\begin{array}{l}12.02 \pm 1.71 \\
(p=0.042)^{*}\end{array}$ & $\begin{array}{l}11.25 \pm 2.13 \\
(p=0.047)^{*}\end{array}$ & $\begin{array}{l}9.28 \pm 1.51 \\
(p=0.09)^{\mathrm{NS}}\end{array}$ \\
\hline Length of the brush border $[\mu \mathrm{m}]$ & $0.82 \pm 0.06$ & $\begin{array}{l}0.41 \pm 0.01 \\
(p=0.041)^{*}\end{array}$ & $\begin{array}{l}0.65 \pm 0.08 \\
(p=0.04)^{*}\end{array}$ & $\begin{array}{c}0.50 \pm 0.02 \\
(p=0.062)^{N S}\end{array}$ & $\begin{array}{c}0.54 \pm 0.07 \\
(p=0.058)^{\text {NS }}\end{array}$ & $\begin{array}{l}0.48 \pm 0.02 \\
(p=1.68)^{\mathrm{NS}}\end{array}$ \\
\hline
\end{tabular}

*Significant; **Highly significant; NS — non significant; DN — diabetic nephropathy; N02-OA — nitro-oleic acid; pre-DN — pre-diabetic nephropathy; pre-DN N02-0A — pre-diabetic nephropathy nitro-oleic acid; post-DN — post-diabetic nephropathy; post-DN-N02-OA - post-diabetic nephropathy nitro-oleic acid. P values for pre-DN Losatan, pre-DN NO2-0A, post -DN losatan and post-DN N02-0A are calculated relative to the DN group. $P$ values for DN group are calculated relative to the control group. Ten randomly chosen, non-overlapping fields $(200 \times)$ was as follows: 0 (none), $1(\leq 10 \%), 2(11-25 \%), 3(26-45 \%), 4(46-75 \%)$, and $5(\geq 76 \%)$.

SBP which proved the development of hypertension with DN. This latter finding coincides with the previous reports of increased incidence of hypertension in patients of DN [18]. Systemic hypertension has been also previously reported to cause intraglomerular hypertension leading to glomerular hypertrophy and injury [17]. Marked obliteration of the glomerular capillary tuft secondary to excessive collagen and mesangial matrix deposition demonstrated in our study readily suggests the occurrence of glomerular hypertension in DN group.

Parathyroid hormone related protein expression in renal tissues and serum angiotensin were also significantly higher in the DN group in the current study. This adds further evidence to the previous reports demonstrating the involvement of PTHrP overexpression in renal injury [6]. Our work also agrees with previous studies that suggested the involvement of PTHrP in the mechanisms related to Angll-induced renal injury $[2,15]$. Furthermore, exogenously administrated Angll, by its AT1R, increases PTHrP expression in glomerular and tubular cells as well as in vascular smooth cells both in vivo and in vitro. Interestingly, PTHrP in the current study showed a strong positive correlation with angiotensin, urea and UAE and negative correlation with GFR. These results are concordant with the previous studies reporting overexpression of PTHrP in the renal tubules and glomeruli in diabetic mice, which was associated significantly with increased UAE [8]. The authors also reported increase in the risk of developing proteinuria in mice with higher PTHrP levels. This also agrees with our results that PTHrP was involved in renal injury associated with DN.
The current study evaluated the possible preventive and therapeutic effects of losartan and NO2-OA on the functional and histopathological parameters of DN.

Administration of losartan as ARB either pre-DN or post-DN showed significant improvement in SBP and fasting serum glucose level compared to diabetic group, with more improvement in pre-DN group. Concordant results have been previously reported [26]. This hypoglycaemic effect of losartan could be due to the effect of losartan in increasing insulin sensitivity and $\beta$-cell responsiveness to glucose and also through enhancement of glucose homeostasis through several insulin signalling pathways [10]. This effect was suggested to occur at the levels of both tyrosine phosphorylation and phosphatidylinositol 3-kinase activation as well as mitogen-activated protein kinase [1].

Our results also showed that pre-DN losartan significantly improved kidney function and was even more effective than post-DN losartan regarding urea, UAE and GFR with a significant difference concerning UAE. These observations are concordant with the previous studies reporting reduction in progression of DN with losartan [24]. Our study has provided an in-depth histopathological analysis of these observations demonstrating increment in tubular epithelial height, improvement in tubular lining with predominance of vesicular nuclei with prominent nucleoli and significant reduction in glomerular and tubular injury markers. Taken together, these data along with the previous histopathological reports [9] provide considerable support for the view that the hallmark features of renal damage including glomerulosclerosis and renal tubular necrosis occur as a result of intense 
intrarenal vasoconstriction subsequent to renin-angiotensin activation [5, 22].

The renoprotective effect of losartan as an ARB may be due to blood pressure reduction and increase in renal blood flow with reduction in intra-glomerular pressure [11]. This was evidenced in the current study by conservation of the glomerular capillary lumina, reversion of the glomerular sclerosis pattern, recession of mesangial proliferation and improvement of vasculopathy in losartan-treated groups relative to their diabetic counterparts.

In the current study, administration of losartan resulted in significant decrease in PTHrP levels. These results support the view that Angll-induced renal damage is associated with renal PTHrP upregulation [6] and that Angll increases PTHrP expression in glomerular and tubular cells both in vivo and in vitro [3].

NO2-OA administration showed a significant decline in fasting serum glucose level in both pre-DN and post-DN groups relative to DN group, with more improvement in pre-DN group. SBP was also improved significantly after pre- and post-DN NO2-OA administration with non-significant difference between both groups. Our data showed improvement in kidney function and glomerular damage parameters only in pre-DN NO2-OA rats versus diabetic rats with non-significant effect with post-DN NO2-OA rats. This could be explained by the actual occurrence of DN pathological changes interfering with maximum beneficial effect of the drug.

NO2-OA has been previously reported to induce non-significant reduction in diabetic glomerulosclerosis [12]. This adds further support to lack of complete resolution of the sclerotic pattern observed in the glomeruli of DN group following NO2-OA administration in our study.

There is a lack of studies concerning the relation of NO2-OA with PTHrP. This work showed a significant reduction in both serum angiotensin and PTHrP after NO2-OA administration with a more significant decline in pre-DN group. This effect may be due to reduction of AT1R mRNA level and gene expression with subsequent angiotensin reduction [23] or due to binding to AT1R, which further reduces heterotrimeric G-protein coupling, and inhibits IP3 (inositol-1,4,5-triphosphate) and calcium mobilisation, without inhibiting Angll binding to the receptor [27].

The current study offers losartan and nitro-oleic acid as promising lines for enhancing renal sparing and maintaining functional integrity of the kidneys in the diabetic patients. The two drugs, especially losartan, can be suggested as prophylactic agents to prevent the deleterious effect of diabetic nephropathy and its undesired consequences of glomerulosclerosis and tubular injury.

Although the results of the current study suggest the superiority of losartan over nitro-oleic acid in ameliorating diabetic nephropathy, yet, the current study used a rat model, and dissimilar outcome in humans could be a possibility. Thus, generalisations cannot be made. Also, in a future study, additional testing with larger sample sizes and/or changes to the dosage of losartan and nitro-oleic acid could more precisely determine the significant difference between the studied groups, especially in the post-diabetic nephropathy groups.

\section{CONCLUSIONS}

Both losartan and NO2-OA were found to improve DN. This study postulated that there is no difference in the preventive effect of both drugs in improving hyperglycaemia and elevated SBP as well as in improving kidney function and tubular injury. However, therapeutic effect of losartan on DN may be more beneficial than NO2-OA effect especially in alleviating diabetes-induced vasculopathy and glomerulosclerosis. Moreover, our data highlight the potential role of Angll antagonist in protection and regression of DN and reduction in PTHrP overexpression supporting the previously reported role of Angll in regulating PTHrP expression.

\section{REFERENCES}

1. Ando K, Fujita T. Anti-diabetic effect of blockade of the renin-angiotensin system. Diabetes Obes Metab. 2006; 8(4): 396-403, doi: 10.1111/j.1463-1326.2005.00526.x, indexed in Pubmed: 16776746.

2. Ardura JA, Rayego-Mateos S, Rámila D, et al. Parathyroid hormone-related protein promotes epithelial-mesenchymal transition. J Am Soc Nephrol. 2010; 21(2): 237-248, doi: 10.1681/ASN.2009050462, indexed in Pubmed: 19959711.

3. Bosch RJ, Ortega A, Izquierdo A, et al. A transgenic mouse model for studying the role of the parathyroid hormone-related protein system in renal injury. J Biomed Biotechnol. 2011; 2011: 290874, doi: 10.1155/2011/290874, indexed in Pubmed: 21052497.

4. Chen YT, Tsai TH, Yang CC, et al. Exendin-4 and sitagliptin protect kidney from ischemia-reperfusion injury through suppressing oxidative stress and inflammatory reaction. J Transl Med. 2013; 11(1): 270, doi: 10.1186/1479-5876-11-270.

5. Davis CL, Gonwa TA, Wilkinson AH. Pathophysiology of renal disease associated with liver disorders: implications for liver transplantation. Part I. Liver Transpl. 2002; 8(2): 
91-109, doi: 10.1053/jlts.2002.31516, indexed in Pubmed: 11862584.

6. Fiaschi-Taesch NM, Santos S, Reddy V, et al. Prevention of acute ischemic renal failure by targeted delivery of growth factors to the proximal tubule in transgenic mice: the efficacy of parathyroid hormone-related protein and hepatocyte growth factor. J Am Soc Nephrol. 2004; 15(1): 112-125, doi: 10.1097/01.asn.0000102470.12285.c6.

7. Gojo A, Utsunomiya K, Taniguchi K, et al. The Rho-kinase inhibitor, fasudil, attenuates diabetic nephropathy in streptozotocin-induced diabetic rats. Eur J Pharmacol. 2007; 568(1-3): 242-247, doi: 10.1016/j.ejphar.2007.04.011, indexed in Pubmed: 17511984.

8. Izquierdo A, López-Luna P, Ortega A, et al. The parathyroid hormone-related protein system and diabetic nephropathy outcome in streptozotocin-induced diabetes. Kidney Int. 2006; 69(12): 2171-2177, indexed in Pubmed: 16783882.

9. Ji Z, Huang C, Liang C, et al. Protective effects of blocking renin-angiotensin system on the progression of renal injury in glomerulosclerosis. Cell Mol Immunol. 2005; 2(2): 150-154.

10. Jin HM, Pan Yu. Angiotensin type-1 receptor blockade with losartan increases insulin sensitivity and improves glucose homeostasis in subjects with type 2 diabetes and nephropathy. Nephrol Dial Transplant. 2007; 22(7): 1943-1949, doi: 10.1093/ndt/gfm049, indexed in Pubmed: 17308317.

11. Kobori $\mathrm{H}$, Mori $\mathrm{H}$, Masaki T, et al. Angiotensin II blockade and renal protection. Curr Pharm Des. 2013; 19(17): 3033-3042, indexed in Pubmed: 23176216.

12. Liu Y, Jia Z, Liu S, et al. Combined losartan and nitro-oleic acid remarkably improves diabetic nephropathy in mice. Am J Physiol Renal Physiol. 2013; 305(11): F1555-F1562, doi: 10.1152/ajprenal.00157.2013, indexed in Pubmed: 23946292.

13. Lorenzo O. Angiotensin II Increases Parathyroid Hormone-Related Protein (PTHrP) and the Type 1 PTH/ /PTHrP Receptor in the Kidney. J Am Soc Nephrol. 2002; 13(6): 1595-1607, doi: 10.1097/01.asn.0000015622. 33198.bf.

14. Noda, M., Katoh, T., Takuwa, N., Synergistic stimulation of parathyroid hormone-related peptide gene expression by mechanical stretch and angiotensin II in rat aortic smooth muscle cells. J Biol Chem. 1994; 269(27): 17911-17917.

15. Park JT, Kang SW. Oxidative stress, the capo of endothelial dysfunction in chronic renovascular hypertension. Kidney Res Clin Pract. 2014; 33(1): 1-2, doi: 10.1016/j. krcp.2014.02.003, indexed in Pubmed: 26877943.

16. Pirola CJ, Wang HM, Kamyar A, et al. Angiotensin II regulates parathyroid hormone-related protein expression in cultured rat aortic smooth muscle cells through transcriptional and post-transcriptional mechanisms. J Biol Chem. 1993; 268(3): 1987-1994, indexed in Pubmed: 8420973.
17. Pyle L. Rapid rise in hypertension and nephropathy in youth with type 2 diabetes: the TODAY clinical trial. Diabetes Care. 2013; 36(6): 1735-1741, doi: 10.2337/ dc12-2420.

18. Raile K, Galler A, Hofer S, et al. Diabetic nephropathy in 27,805 children, adolescents, and adults with type 1 diabetes: effect of diabetes duration, A1C, hypertension, dyslipidemia, diabetes onset, and sex. Diabetes Care. 2007; 30(10): 2523-2528, doi: $10.2337 / \mathrm{dc07}-0282$, indexed in Pubmed: 17630266.

19. Romero M, Ortega A, Olea N, et al. Novel role of parathyroid hormone-related protein in the pathophysiology of the diabetic kidney: evidence from experimental and human diabetic nephropathy. J Diabetes Res. 2013; 2013: 162846, doi: 10.1155/2013/162846, indexed in Pubmed: 23984429.

20. Singh R, Singh AK, Leehey DJ. A novel mechanism for angiotensin II formation in streptozotocin-diabetic rat glomeruli. Am J Physiol Renal Physiol. 2005; 288(6): F1183-F1190, doi: 10.1152/ajprenal.00159.2003, indexed in Pubmed: 15701818.

21. Tang SCW, Chan GCW, Lai KN. Recent advances in managing and understanding diabetic nephropathy. F1000Res. 2016; 5, doi: 10.12688/f1000research.7693.1, indexed in Pubmed: 27303648.

22. Wadei HM, Mai ML, Ahsan N, et al. Hepatorenal syndrome: pathophysiology and management. Clin J Am Soc Nephrol. 2006; 1(5): 1066-1079, doi: 10.2215/CJN.01340406, indexed in Pubmed: 17699328.

23. Wang W, Li C, Yang T. Protection of nitro-fatty acid against kidney diseases. Am J Physiol Renal Physiol. 2016; 310(8): F697-F704, doi: 10.1152/ajprenal.00321.2015, indexed in Pubmed: 26719362.

24. Weil EJ, Fufaa G, Jones LI, et al. Effect of losartan on prevention and progression of early diabetic nephropathy in American Indians with type 2 diabetes. Diabetes. 2013; 62(9): 3224-3231, doi: 10.2337/db12-1512, indexed in Pubmed: 23545707.

25. Welsch S, Schordan E, Coquard C, et al. Abnormal renovascular parathyroid hormone-1 receptor in hypertension: Primary defect or secondary to angiotensin ii type 1 receptor activation? Endocrinology. 2006; 147(9): 4384-4391, doi: 10.1210/en.2005-1517, indexed in Pubmed: 16728497.

26. Yazgan ÜC, Taşdemir E, Bilgin HM, et al. Comparison of the anti-diabetic effects of resveratrol, gliclazide and losartan in streptozotocin-induced experimental diabetes. Arch Physiol Biochem. 2015; 121(4): 157-161, doi: 10.3109/1 3813455.2015.1062898, indexed in Pubmed: 26161595.

27. Zhang J, Villacorta L, Chang L, et al. Nitro-oleic acid inhibits angiotensin II-induced hypertension. Circ Res. 2010; 107(4): 540-548, doi: 10.1161/CIRCRESAHA.110.218404, indexed in Pubmed: 20558825. 